\title{
Highly Sensitive Detection of Anthracene at the Interface Region of a Hydrocarbon-Water Two-Phase System Using Laser Two-Photon Ionization
}

\author{
Yao Qun Li*, Takanori INOUE** and Teiichiro OGAWA** \\ *SEDC Laboratory of Analytical Sciences, Department of Chemistry, Xiamen University, \\ Xiamen 361005, P.R. China \\ **Department of Molecular Science and Technology, Kyushu University, \\ Kasuga, Fukuoka 816, Japan
}

\begin{abstract}
The laser two-photon ionization technique has been applied to an interface region in a two-phase system. Anthracene, which stays preferentially in the hydrocarbon phase, could be determined using laser irradiation through the water phase. The detection limit $(S / N=3)$ was $1.8 \times 10^{-9} \mathrm{M}$.
\end{abstract}

Keywords Laser, highly sensitive detection, photoionization, two-phase system, interface, anthracene

Laser two-photon ionization combined with conductometry offers a convenient and selective method for the detection of photoabsorbing molecules in the presence of a large amount of transparent solvent or bulk molecules. The technique has been successful in the highly sensitive detection in nonpolar solvents ${ }^{1-4}$, on a metal surface ${ }^{5,6}$ and on a water surface. ${ }^{7-12}$ Recently, surface photoionization has been applied to an aqueous flow in order to indicate the analytical potential of this technique for a flow detector. ${ }^{13}$

The successes on surfaces have stimulated us to apply this technique to a solution interface. As a first step in an interface study we applied this technique to a hydrocarbon-water two-phase system. This approach was successfully used for a highly sensitive detection of anthracene at the interface region of the two-phase system.

\section{Experimental}

The experimental apparatus was similar to that 8,9 employed for detection on a water surface, except for the photoionization cell as shown in Fig. 1. The third harmonic of a Nd:YAG laser (Spectra Physics GCR-11; $60 \mathrm{~mJ}$ and $10 \mathrm{~ns}$ at $355 \mathrm{~nm}$ ) was operated at a repetition rate of $5 \mathrm{~Hz}$, and was focused softly into the interface through the water phase with an incident angle of $85^{\circ}$ to the interface normal. Most of the laser beam arriving

Y.Q.L. present address: Department of Molecular Science and Technology, Kyushu University, Kasuga, Fukuoka 816, Japan. from below was reflected at the interface, while part of it passed through the interface. The cell comprised a fluorescence cell $(4 \times 1 \times 1 \mathrm{~cm})$ and a pair of copper electrodes $(4 \times 4 \mathrm{~mm})$. The electrode spacing was $8 \mathrm{~mm}$. The upper electrode was connected to a high-voltage supply (Ikegami, HD2.5K-M) through a current-limit resistor $(10 \mathrm{k} \Omega)$. The photoionization current was measured through the lower electrode with a current amplifier (Keithley 428) and an oscilloscope (DCS-8200, $50 / 60 \mathrm{~Hz}$ ).

Anthracene (Guaranteed Reagent) was used as purchased. Hexane was distilled and dehydrated with $\mathrm{CaCl}_{2}$. Heptane, cyclohexane, 2,2,4-trimethylpentane

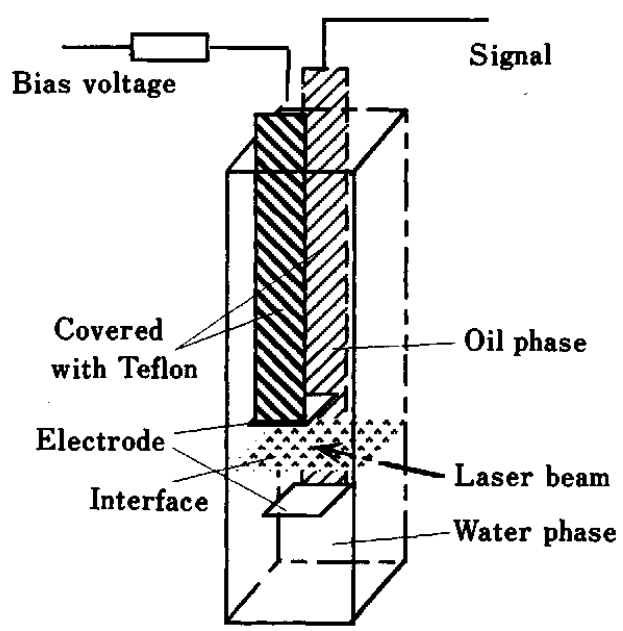

Fig. 1 Schematic diagram of the cell. 


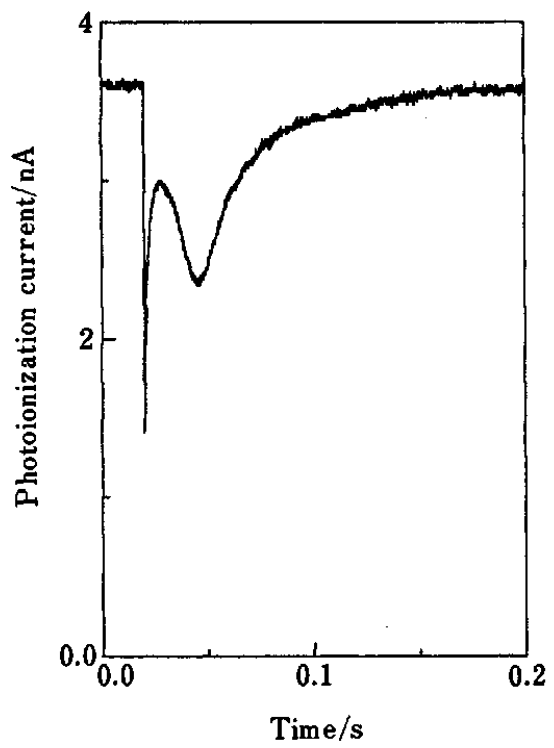

Fig. 2 Time profile of the photoionization current of anthracene $\left(10^{-6} \mathrm{M}\right.$ in heptane). Laser pulse energy: $1.6 \mathrm{~mJ}$.

and benzene (all Special Reagent) were used as supplied. The water was distilled and deionized. The indicated concentrations of anthracene were those prepared in organic solvents.

\section{Results and Discussion}

When anthracene is dissolved in a hydrocarbon-water two-phase system, anthracene stays preferentially in the hydrocarbon phase. When a laser irradiated the sample at the interface through water, as in the present study, part of the laser beam proceeded through the interface into the hydrocarbon phase. Because the efficiency of photoionization is proportional to the square of the laser intensity, however, the efficiency of photoionization is much larger at the interface. The "interface region" concerned in the present study is a region along the borderline of hydrocarbon and water phases where photoionization proceeds.

A typical time profile of the current produced by laser two-photon ionization of anthracene at the heptanewater interface region is shown in Fig. 2. The profile consists of a fast component and a slow component. Both negative and positive bias voltages offered essentially an identical time profile, though the sign of the current was opposite. The initial decrease in the photoionization current was due to a volume recombination of the cations and anions. The time of the second peak shifted with the incident laser power and the concentration of anthracene, which exhibits an inhomogeneous space charge effect on the profile. ${ }^{14}$

The area of the time profile (photoionization charge) was not linearly proportional to the current (the height of the fast peak), as shown in Fig. 3. The data were

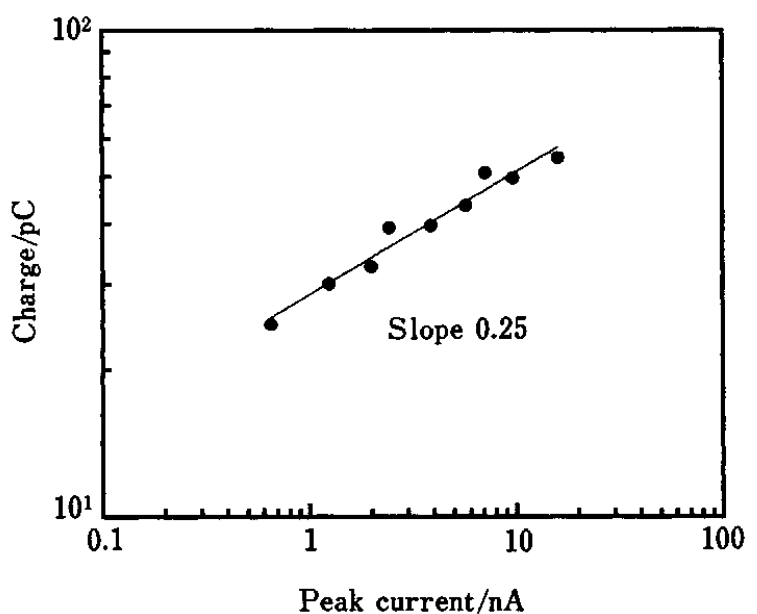

Fig. 3 Relationship between the photoionization charge area and the peak current. Laser pulse energy: 0.52, 0.90, $1.6 \mathrm{~mJ}$. Concentration of anthracene in heptane: $4 \times 10^{-7}$, $4 \times 10^{-6}, 10^{-5} \mathrm{M}$.

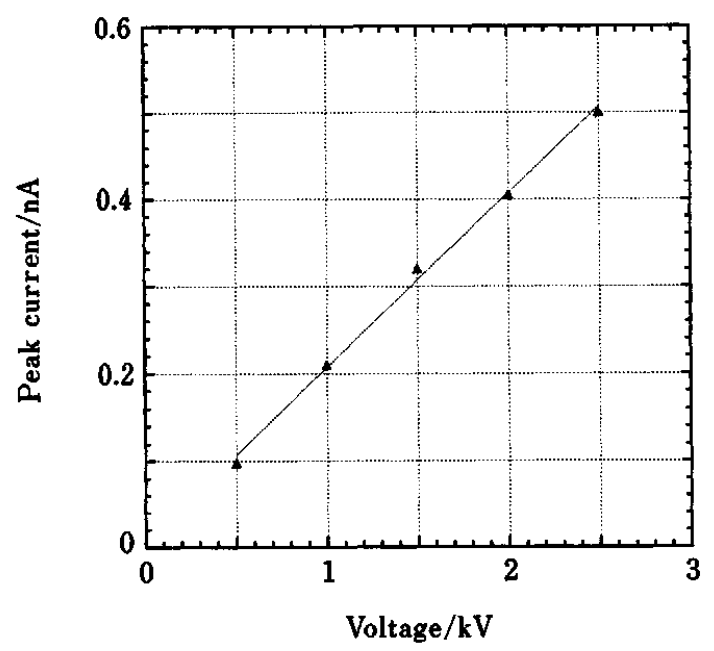

Fig. 4 Dependence of the photoionization peak current on the bias voltage for anthracene $\left(10^{-7} \mathrm{M}\right.$ in heptane). Laser pulse energy: $1.6 \mathrm{~mJ}$. Negative bias voltage.

obtained by changing both the laser power and the concentration of anthracene; they exhibited a slope of 0.25 on a $\log -\log$ plot. This finding indicated that the apparent photoionization charge did not increase as much as the current, due to strong charge recombination.

The photoionization peak current was linearly proportional to the applied negative bias voltage (Fig. 4), although the relation was not linear for a positive bias voltage. A voltage of $2 \mathrm{kV}$ was applied for the following measurements.

Two-photon ionization of anthracene was carried out for four hydrocarbons and water two-phase systems. The photoionization peak current and the leakage currents are summarized in Table 1. Although the 
Table 1 Comparison of leakage current of solvents and peak current of photoionization for anthracene $\left(4 \times 10^{-7} \mathrm{M}\right)$

\begin{tabular}{lcccc}
\hline & $\begin{array}{c}\text { Heptane// } \\
\text { water }\end{array}$ & $\begin{array}{c}\mathrm{TMP}^{\mathrm{a}} / \\
\text { water }\end{array}$ & $\begin{array}{c}\text { Hexane/ } \\
\text { water }\end{array}$ & $\begin{array}{c}\text { Benzene/ } \\
\text { water }\end{array}$ \\
\hline Leakage current/nA & $<0.0005$ & 0.16 & 0.03 & 0.38 \\
Peak current/nA & 0.50 & 14.4 & 1.13 & 1.95 \\
\hline
\end{tabular}

a. 2,2,4-Trimethylpentane.

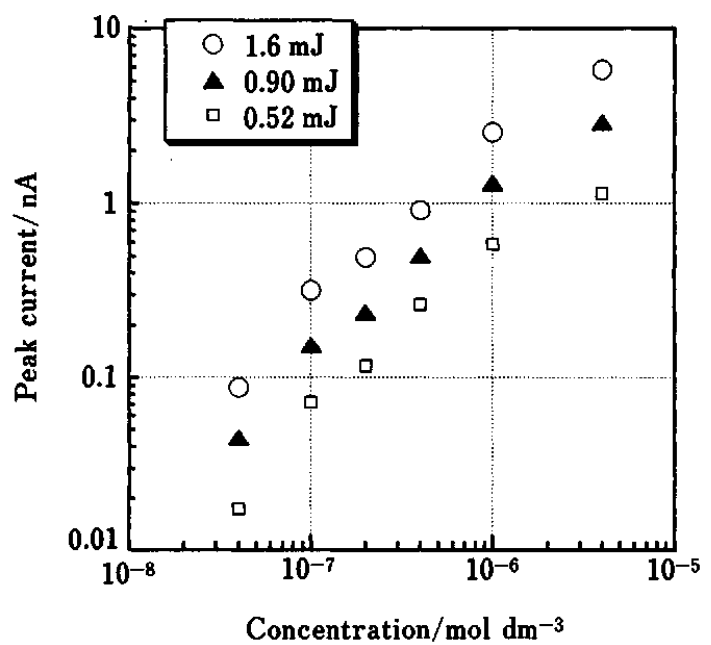

Fig. 5 Concentration dependence of the peak current of anthracene at three laser pulse energies. Negative bias voltage.

signal of anthracene at the heptane/water interface region was smallest, the leakage current of the system was smallest and the signal-to-noise ratio was the largest. Thus, heptane was used for determining the detection limits.

The analytical curves of anthracene were linear for three laser pulse energies of $0.52,0.90,1.6 \mathrm{~mJ} /$ pulse (Fig. 5). When a positive bias voltage was applied, the linearity was slightly poor. The detection limit was obtained as the concentration at which the ratio of a signal to noise was 3 at a laser pulse energy of $0.90 \mathrm{~mJ}$. The blank signal of the heptane/water system was $15.7 \mathrm{pA}$, and its standard deviation was $0.68 \mathrm{pA}$. The detection limit was $1.8 \times 10^{-9} \mathrm{M}$.

The present study has indicated that laser photo- ionization combined with conductometry is a sensitive and convenient method to detect photoabsorbing molecules at the interface region in a two-phase system. Anthracene, which stays preferentially in the hydrocarbon phase, can be determined using laser irradiation through the water phase. Laser photoionization is thus a potential tool for investigating the phenomena of a two-phase system. A further investigation of the interface of a two-phase system is proceeding.

A JSPS postdoctoral fellowship of Japan Society for the Promotion of Science to YQL is gratefully acknowledged. The authors wish to thank Miss Miki Sato for her assistance in the experiment and Dr. Keiji Nakashima for useful discussions.

\section{References}

1. T. Ogawa, K. Kise, T. Yasuda, H. Kawazumi and S. Yamada, Anal. Chem., 64, 1217 (1992).

2. E. Voigtman and J. D. Winefordner, Anal. Chem., 54, 1834 (1982).

3. S. Yamada, S. Sato, H. Kawazumi and T. Ogawa, Anal. Chem., 59, 2719 (1987).

4. T. Ogawa, M. Sato, M. Tachibana, K. Ideta, T. Inoue and K. Nakashima, Anal. Chim. Acta, 299, 355 (1995).

5. T. Ogawa, T. Yasuda and H. Kawazumi, Anal. Sci., 8, 81 (1992).

6. T. Ogawa, T. Yasuda and H. Kawazumi, Anal. Chem., 64, 2615 (1992).

7. H. Chen, T. Inoue and T. Ogawa, Anal. Chem., 66, 4150 (1994).

8. K. Masuda, T. Inoue and T. Yasuda, K. Nakashima and T. Ogawa, Anal. Sci., 9, 297 (1993).

9. T. Inoue, K. Masuda, K. Nakashima and T. Ogawa, Anal. Chem., 66, 1012 (1994).

10. T. Ogawa, H. Chen, K. Masuda and T. Inoue, Anal. Sci, 10, 219 (1994).

11. T. Ogawa, H. Chen, T. Inoue and K. Nakashima, Chem. Phys. Lett., 229, 328 (1994).

12. T. Ogawa, H. Chen, T. Inoue and K. Nakashima, Anal. Sci., 10, 849 (1994).

13. T. Ogawa, S. Sumi and T. Inoue, Instrum. Sci. Technol, 23, 311 (1995).

14. K. Nakashima, M. Kise and T. Ogawa, Chem. Lett., 1992, 837.

(Received May 9, 1996)

(Accepted July 12, 1996) 